\title{
Survey of Wireless Geolocation Techniques
}

\author{
A. Roxin, J. Gaber, M. Wack, A. Nait-Sidi-Moh \\ Laboratoire Systèmes et Transports (SeT) \\ Université de Technologie de Belfort-Montbéliard (UTBM)
}

\author{
90010 Belfort, France
}

ana.roxin@utbm.fr

\begin{abstract}
Ubiquitous and pervasive networks and applications include a growing number of research themes. In most use cases, applications require location information to interpret their environment and behave accordingly. In this paper, location algorithms and positioning methods that can be used for wireless geolocation are presented.
\end{abstract}

Index Terms-Wireless geolocation, localization technique and algorithms, AOA, TDOA, OTDOA, UTDOA, RSS, GPS, A-GPS, pervasive computing, ubiquitous computing.

\section{INTRODUCTION}

Locating mobile devices has always been a critical problem. It becomes even more critical today, as the number of context-aware applications is continuously growing. Acquiring the location information of a mobile device allows providing more value-added applications. Although GPSbased location is reliable and accurate for outdoor situations, it does not provide acceptable performance in indoor and urban areas. Other technologies have been developed with the aim of implementing the specificities of ubiquitous and pervasive environments. In this report, main algorithms and positioning techniques used for wireless geolocation are presented.

\section{WIRELESS GEOLOCATION SYSTEM}

A wireless geolocation system has three major components (Figure 1):

- The location sensing device that allows determining the relative position of the mobile device,

- The positioning algorithm that computes the metrics reported by the location sensing device, in order to estimate the position of the mobile device,

- The display system, which displays the computed position of the mobile device.

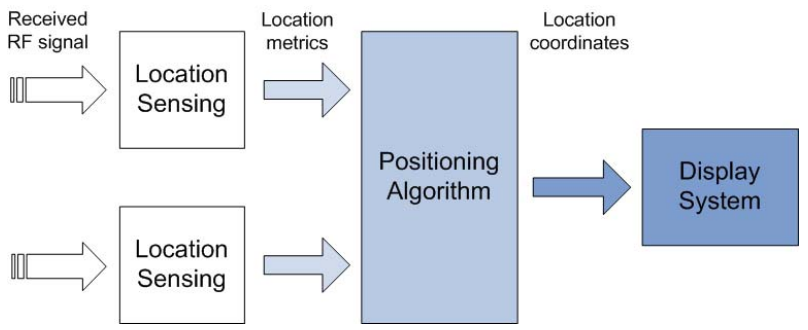

Figure 1. A wireless geolocation system

Two categories of localization techniques have been isolated:

- Dedicated techniques - methods using a dedicated infrastructure (IR, RF, etc.)

- Non dedicated techniques - methods using non dedicated systems

Only non dedicated methods are discussed in this paper, since they suit best for ubiquitous and pervasive environments. Dedicated methods provide a high degree of accuracy, but need expensive, energy-consuming and scale-limited equipments. One may found various papers describing existing dedicated methods [1-5].

The rest of the paper is organized as follows. In section III, localization algorithms are presented. In section IV, positioning methods are described. A conclusion is given in section V. Section VI provides definitions for all the acronyms cited in the text.

\section{LOCALIZATION ALGORITHMS}

In this section, algorithms used by non-dedicated localization techniques are described. To ease the understanding, first are reviewed fundamental principles employed by these techniques. Then an introduction to recent researches will be made.

\section{A. Principles}

There are two fundamental principles used in traditional localization techniques: triangulation and trilateration. 


\section{1) Trilateration}

This method uses the distance between a BS and the mobile device. It requires 3 BSs. The position of the mobile device is determined using an RF location sensing technique.

The mobile device is located by the intersection point of 3 circles. Each circle has for radius the distance between the mobile device and the considered BS as illustrated in Figures $2 \mathrm{a}$ and $2 \mathrm{~b}$.

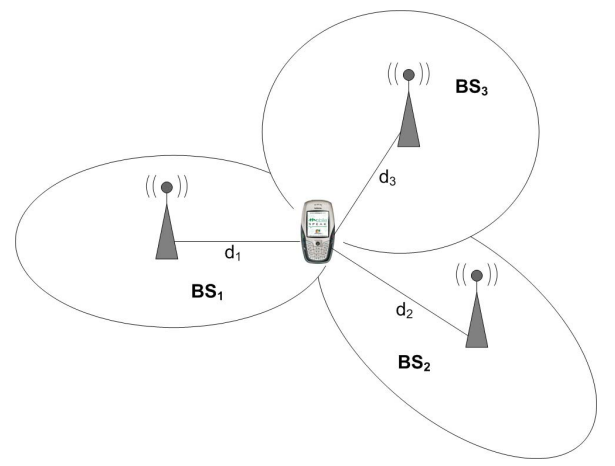

Figure 2a. Locating the mobile device by the intersection of three circles with radii $d_{1}, d_{2}$ and $d_{3}$.

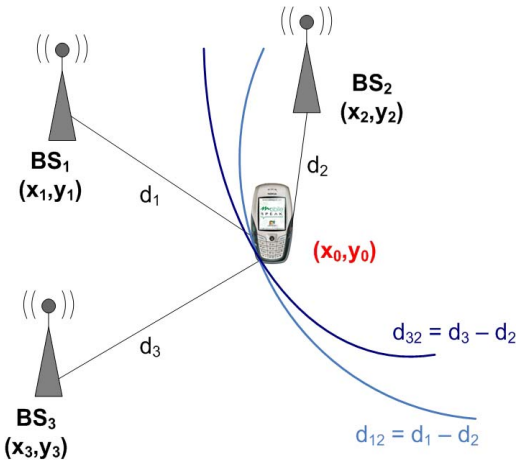

Figure 2b. Locating the mobile device by performing a TDOA.

\section{2) Triangulation}

This method is based on the estimation of the direction of arrival of a signal from the mobile device that has to be located. AoA (presented in section 4.1.4.) is an example of a positioning method that uses triangulation.

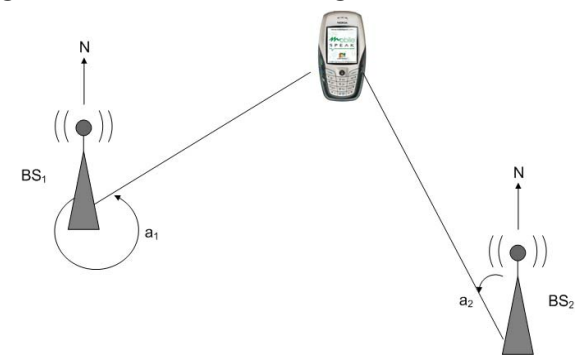

Figure 3. Estimation of the distance between a mobile device and the BS, using AOA.

\section{B. Radio map}

The principle of the radio map is to build a signal strength model. The location of the mobile device is estimated by finding a best match from the signal strength model.

This principle comprises two:

- The offline phase when the radio map is built. During this phase, RF signals are collected from several predefined access points.

- The online phase when the mobile device location is estimated. During this phase, a location algorithm is used to find a matching location, starting from the measured RSS.

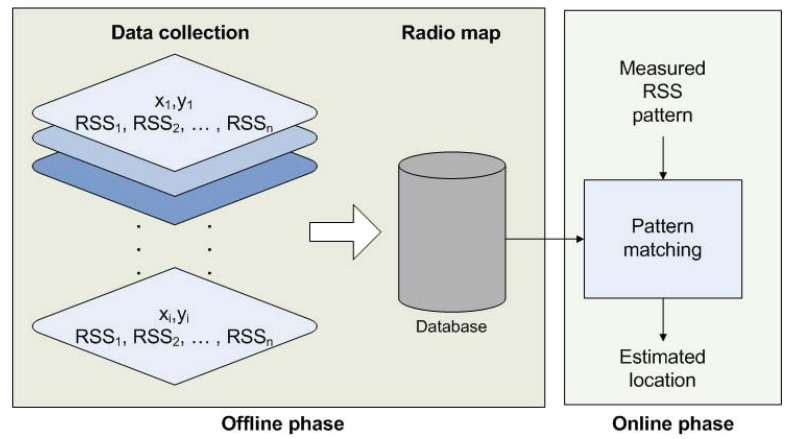

Figure 4. Radio map.

During the online phase, a localization algorithm is used to locate the mobile device. These algorithms are divided into two categories:

- Algorithms using deterministic techniques.

- Algorithms using probabilistic techniques.

These two categories will be presented in the rest of this section.

\section{1) Deterministic techniques}

Deterministic techniques use scalar values of measured RSS from Access Points (AP). The measured values are averaged into a given location, which is associated to the location of the considered mobile device.

\section{a) Nearest Neighbor in Signal Space (NNSS)}

This method computes the distance between the RSS measured by the mobile device and all the other RSS in the radio map. Mobile device location is estimated as the minimal distance computed, which is the smallest Euclidean distance.

b) Nearest Neighbor in Signal Space - Average (NNSS$A V G)$

This method is similar to the NNSS method. Instead of choosing only one neighbour, it picks several nearest neighbours in signal space. The idea is to choose $\mathrm{k}$ nearest 
neighbours and compute the mobile device location by averaging the coordinates of the $\mathrm{k}$ neighbours.

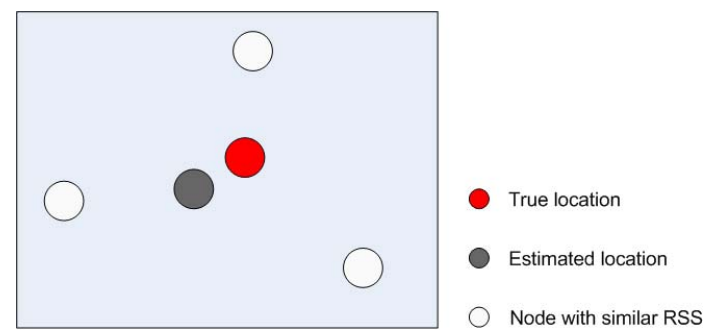

Figure 5. NNSS-AVG method

c) Smallest polygon

This method is similar to the NNSS-AVG method, but instead of using the signal domain, it uses the space domain.

Several nearest neighbours are selected (using various methods), and various polygons are formed. The area of each polygon is then estimated. The algorithm selects the polygon with the smallest area. The location of the mobile device is then estimated as the centre of the previously selected polygon.

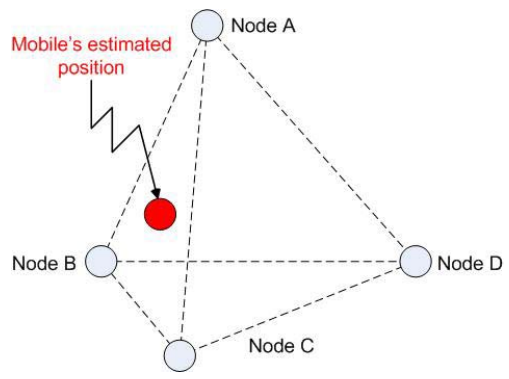

Figure 6. Smallest polygon method - candidate locations (A, B, C and $\mathrm{D})$ form various triangles. The centre of $\triangle \mathrm{ABC}$ is the estimated location, since it has the smallest area.

d)

Approximate Point-In-Triangulation (APIT)

This algorithm is often applied to wireless sensor environments. Anchors are selected among the sensing nodes of a wireless sensor network.

Each anchor broadcasts beacons with location information about the anchor position. A node receives beacons from a set of $\mathrm{N}$ audible anchors. It then builds triangles from the $\mathrm{N}$ audible anchors. The mobile device tests whether it is inside or outside each triangle. This step is called the APIT testing. The area where the most triangles overlap is the most probable area for the location of the mobile device. This is the APIT aggregation [6]. A SCAN approach is used to determine the maximum area where the mobile device is located. Once this area is determined, its centre of gravity is calculated and considered as the estimated location of the mobile device.

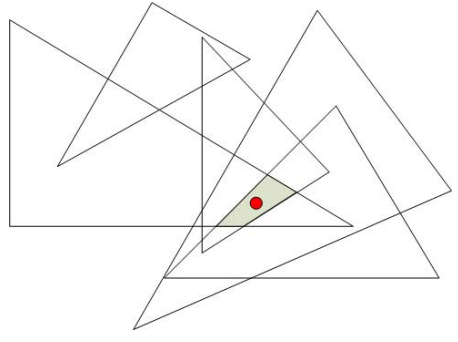

Figure 7. Overview of the APIT algorithm.

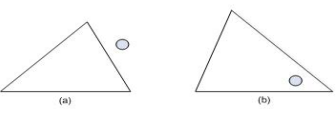

Figure 8. (a) A node is inside the triangle. (b) A node is outside the triangle.

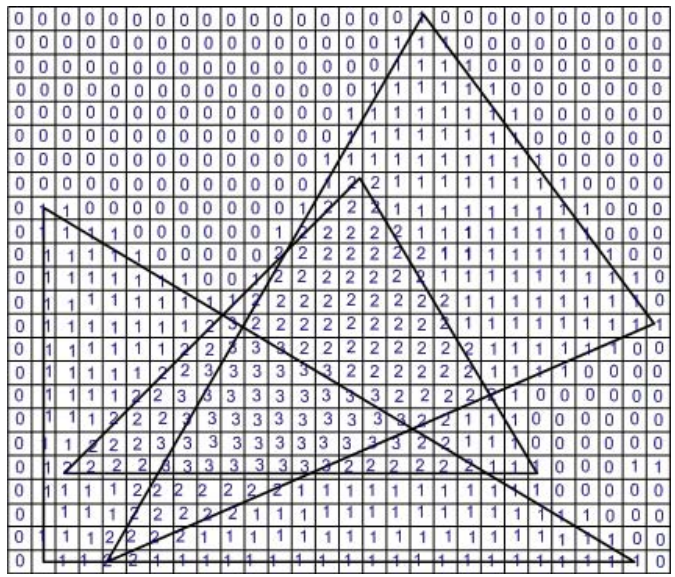

Figure 9. Using SCAN approach to execute APIT aggregation.

\section{2) Probabilistic techniques}

Probabilistic techniques use RSS distributions received from every Access Point (AP). RSS are values that vary in time, as they depend on environments factors [8-11].

The main idea is to assume that RSS distributions are similar to Gaussian probability distributions. During the offline phase, for each AP, a mean and a variance value are computed for the received RSS distribution. Probabilities are then computed for each predefined location, according to the radio map. The most probable location is estimated as the location of the considered mobile device. 


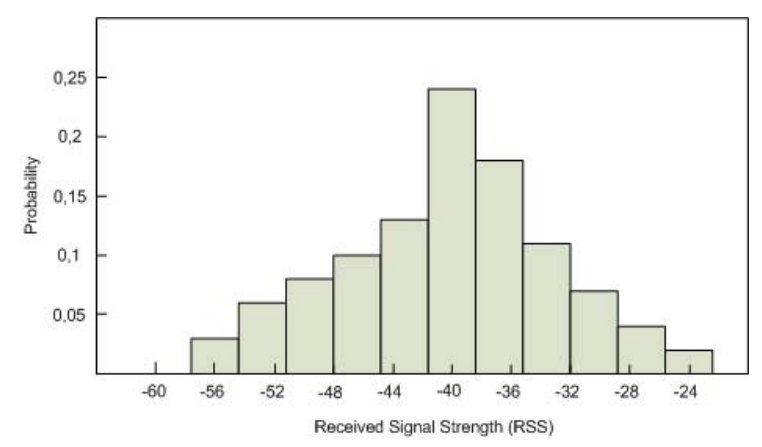

Figure 10. The RSS values received from access point at a fixed location vary with time.

\section{Reference point}

This algorithm allows locating a mobile device in a realtime environment. Several studies have shown that RSS from a given fixed location vary over time [8-11]. Radio maps store RSS values obtained during the offline phase, and with varying RSS values, computed location may be false.

The reference point method uses reference tags, or RFID tags. There are two types of active RFID tags:

- Reference tags - Reference tags are the active RFID tags used for calibrating environmental parameters.

\section{- Tracking tags - RFID tags associated to tracked objects.}

In order to estimate the distance between a transmitter (i.e., tagged object or reference tag) and a receiver (i.e., RFID reader), several methods exist, going from Euclidean distance calculus to probabilistic models using log-distance path loss models.

In [7] Yun et al. present a triangulation technique that uses regression. Starting from RFID signals and pre-computed regression equations, the estimated distances from the tracking tag to the RFID readers, $l_{1}, l_{2}$ and $l_{3}$, are obtained. The estimated distances from the regressive equations of three readers allow computing the estimated location $(u, v)$ of the tag.

\section{Path loss}

Path-loss models allow calculating the distance between a mobile device and 3 APs, with known locations. Various pathloss models exist. Generally, path-loss models use trilateration to determine the location of the mobile device $[12,13]$.

\section{Positioning METHODS}

Now that the main algorithms have been presented, a rundown of the most popular methods of position location will be made. Conventional positioning techniques rely on the angle of arrival (AOA), received signal strength (RSS), the time of arrival (TOA) and the time difference of arrival (TDOA) measurements [14].

Each technique has its own merits and drawbacks, under given cost and complexity constraints. In this section, conventional positioning techniques are presented, starting with network-based techniques and moving into handset-based methods.

\section{A. Network-based methods}

\section{1) CellID (CID)}

Cell Identification (CID) is the process of using network's knowledge of the mobile device, within the controlling cell site and communicating the sector information. The geographical centre of this area provides a rough estimate of the location of the caller (Figure 12).

CID operates in GSM, GPRS and UMTS networks. It requires the network to identify the BTS to which the cell phone is communicating and the location of that BTS.

The accuracy of this method depends on the cell size, and can be very poor in many cases (the GSM cell diameter is between $2 \mathrm{Km}$ to $20 \mathrm{Km}$ ).

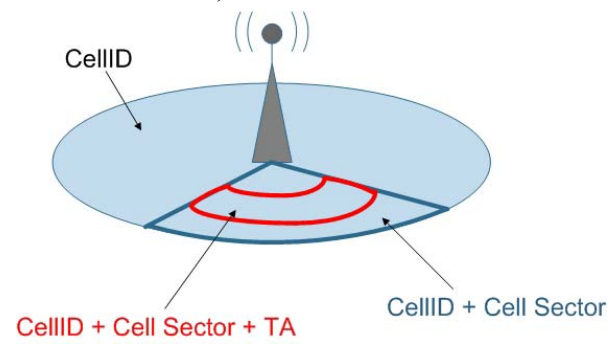

Figure 12. CID principle.

\section{2) Received Signal Strength (RSS)}

The distance between two nodes can be estimated by measuring the energy of the received signal at one end. This distance-based technique requires at least three reference nodes to determine the two-dimensional location of a given node. This technique uses a triangulation approach to determine the location of the mobile unit, as depicted in Figure 13. The dashed circles indicate errors in RSS measurements, [26]. 


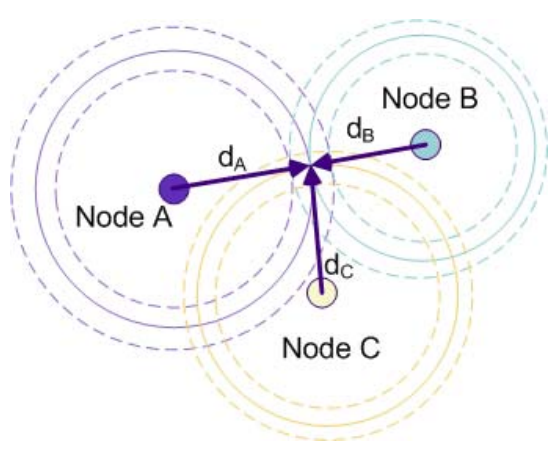

Figure 13. Illustration of positioning via the triangulation of three RSS measurements from nodes A, B and C.

RSS systems are very interesting for urban and indoor geolocation systems, given that this technique is already available for cellular and WLAN networks, without any further changes.

But, a direct measurement of the distance from the RSS cannot be reliable, since the value of the RSS mainly depends on the path-loss model that has been considered. Besides, RSS measurements depend on the channel characteristics. Therefore, RSS-based positioning algorithms are sensitive to channel parameters estimation.

\section{3) Multipath/Fingerprint}

A wireless signal bounces off a variety of solid objects on the way to its destination, causing multipath interference. The same signal is received multiple times due to the delay caused by bouncing off objects and taking different paths to the destination (Figure 14).

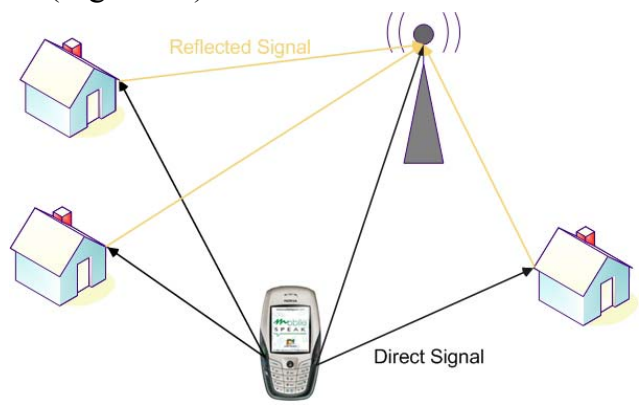

Figure 14. Multipath / Fingerprint - the sum of all signals creates a « fingerprint » signal that is stored and indexed.

Multipath fingerprinting takes advantage of this characteristic to describe signals that are received from certain locations. To employ this system, an operator must send test units around to various locations so the BSs can record the fingerprints and create a database for comparison later on. Of course, if new construction occurs in an area, the fingerprint will change and must be re-recorded.

\section{4) Angle Of Arrival (AOA)}

An AOA-based positioning technique involves measuring angles of the node seen by reference nodes [14]. In order to determine the location of a mobile device in a twodimensional space, it is sufficient to measure the angles of the straight lines that connect the node and two reference nodes, as shown in Figure 15 [25].

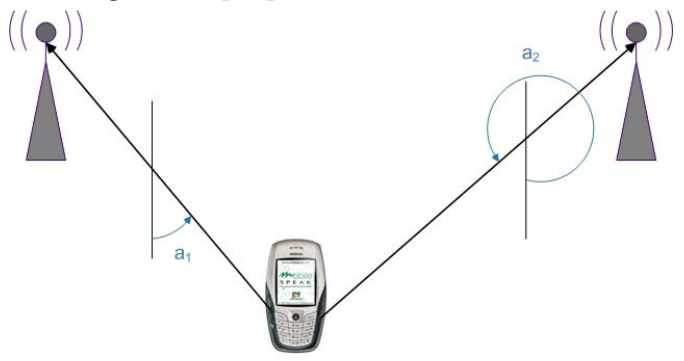

Figure 15. Angle Of Arrival

This method uses multiple antennas at a BS to determine the incident angle of an arriving signal. If a handset transmitting a signal is within LOS, the antenna array can determine what direction the signal is coming from. A second BS with the same technology must also locate the mobile device and compare the obtained position with data from the first BS $[15,16]$.

AOA systems must be designed to account for multipath signals, since they may confuse the location of the handset. Also, installing and aligning antenna arrays on BSs can be a sensitive and costly process $[14,26]$.

\section{5) Time Of Arrival (TOA)}

Time-based positioning techniques rely on measurements of signal travel times between nodes. TOA is a technique that allows locating a mobile device by calculating the time of arrival of the signal from the mobile to more than one BS [14].

The TOA can be estimated either by measuring the phase of the received narrowband carrier signal or by direct measurement of the arrival time of a wideband narrow pulse [26]. The first solution cannot provide an accurate estimation of location in heavy multipath environments. For those environments, the second solution is best suited. Indeed, the second solution allows lowering the distance measurement error when increasing bandwidth. This means that for a UWB environment the distance error caused by erroneous estimates of the TOA will be quite insignificant, if using a direct measurement for a wideband narrow pulse.

If two nodes have a common clock, the node receiving the signal can estimate the TOA of the incoming signal, which is time-stamped by the reference node. Therefore, this method requires the cellular network to be synchronized.

TOA is a more accurate technique than CID. Still the cost and overhaul of a network, required to implement TOA may be disproportionate in relation to the resulting accuracy enhancement, unless service providers supply their own overlay service to attach to a network [26].

\section{6) Time Difference Of Arrival (TDOA)}

The TDOA technique can be employed when there is no synchronization between a given node and the reference nodes, but there is synchronization between reference nodes $[14,26]$. 
The TDOA of two signals travelling between the given node and two reference nodes is estimated [17]. This determines the location of the given node on a hyperbola with foci at the two reference nodes. A third reference node is needed to triangulate the position of the mobile unit (Figure 16). To achieve accurate positioning, the reference nodes must be synchronized in time. If there isn't a common clock between the nodes, RTT between two transceiver nodes can be measured to estimate the distance between nodes $[19,20]$.

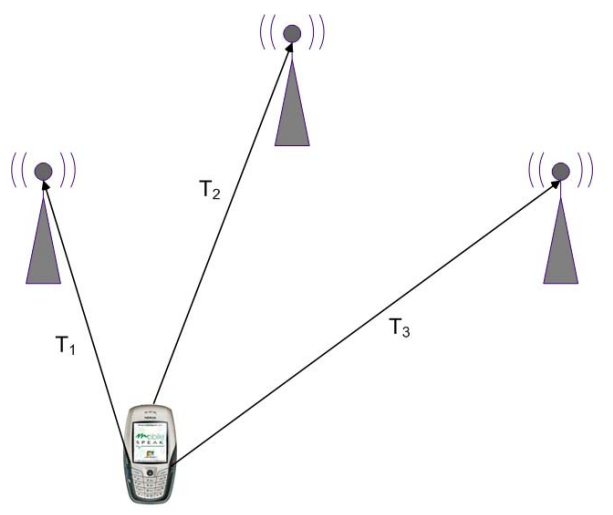

Figure 16. TDOA - Comparing time differences $\left(T_{1}-T_{2}, T_{2}-T_{3}, T_{1}\right.$ $-\mathrm{T}_{3}$ ) locates the mobile unit.

\section{7) Observed Time Difference Of Arrival (OTDOA)}

OTDOA operates only on UMTS networks. The OTDOA LS estimates the position of a mobile device by referencing signal reception time at the $\mathrm{MS} / \mathrm{UE}$ from a minimum of 3 Node B stations. The mobile's position is at the intersection of at least two hyperbolas defined by the OTDAs of the UMTS frames from multiple Node B stations, as shown in Figure 17.

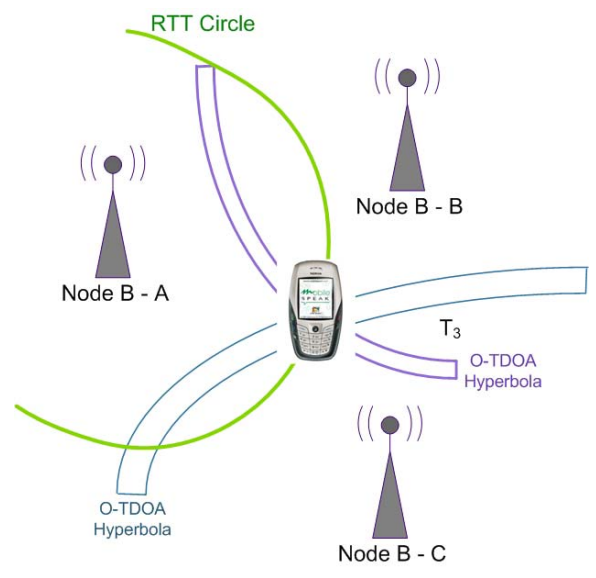

Figure 17. OTDOA technique

\section{8) U-TDOA (Uplink Time Difference of Arrival)}

U-TDOA is a positioning method developed by TruePosition [22], and which relies upon multi-lateration. As it is completely network-based, no additional chip or software needs to be installed into the mobile device.

U-TDOA determines a mobile phone's location by comparing the times at which a cell signal reached the LMUs installed at the operator's BTSs (Figure 18). Accuracy is determined by the network layout and deployment density of LMUs to BTSs.

U-TDOA technology works very well in urban, suburban and indoor environments - suffering only in extreme rural conditions [21].

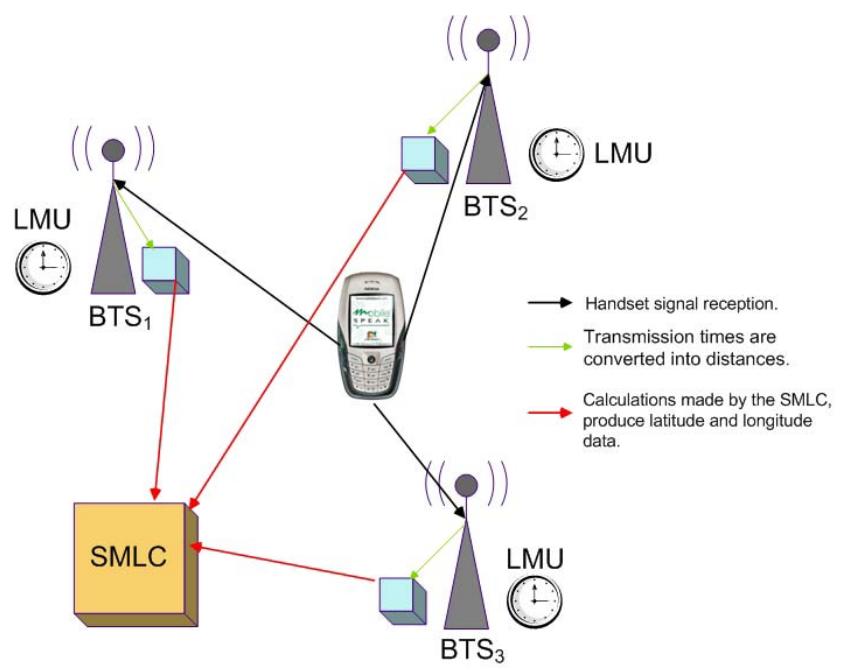

Figure 18. U-TDOA technique

\section{B. MS-based methods}

\section{1) Global Positioning System (GPS)}

Largely considered the most accurate location technology, GPS uses satellites to fix the position of a mobile unit. The mobile unit needs special hardware and software for receiving GPS signals [24].

The GPS has 3 parts:

- The space segment, which consists of 24 satellites.

- The user segment, which consists of receivers.

- The control segment, which consists of monitor stations that make sure the satellites are working properly.

Satellites are equipped with very precise clocks that keep accurate time to within $3 \mathrm{~ns}$ (nanoseconds). Precision timing is important because the receiver must determine exactly how long it takes for signals to travel from each GPS satellite.

The added cost of components in the handset has made this a less desirable option in the past, but prices are coming down to make it more attractive for lower-end handsets. GPS also suffers from a relatively long delay (sometimes more than a minute) to get an initial fix on the location of a mobile unit.

\section{2) Assisted GPS (A-GPS)}

A-GPS systems are set up to resolve the long delay that can occur in locating a mobile unit when using GPS. Data about the mobile unit is transmitted through the network of BSs to speed up the location process, bringing it down to only a few seconds. This occurs when obstructions block the view from a handset to a GPS satellite [23]. 
Wireless A-GPS operates on GSM, GPRS and UMTS networks. Like GPS, A-GPS uses satellites in space as reference points to determine location.

A-GPS can be accurate up to 10 meters.

A-GPS provides better accuracy than CID, E-OTD or OTDOA, and operates on asynchronous or synchronous networks without the need for LMUs (although LMU information can be used if it is available) [21].

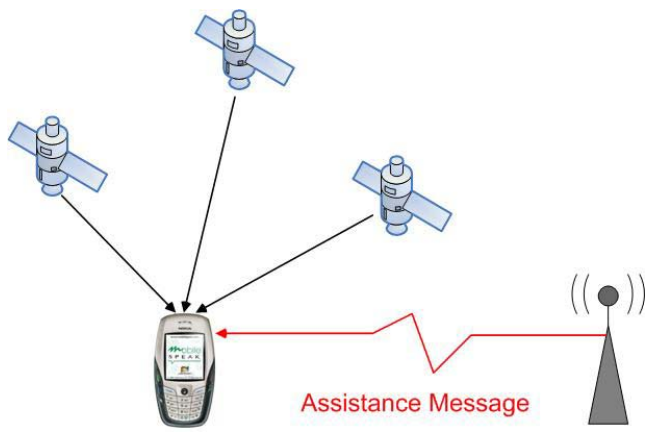

Figure 19. A-GPS Principle

An A-GPS implementation has almost negligible impact on the infrastructure and can easily support roaming, but requires A-GPS circuitry inside the phone, so legacy handsets cannot be supported without modification. A-GPS requires message exchanges with an A-GPS LS in the infrastructure, but this is handled in a flexible manner and exchanged messages are small-sized [24].

\section{3) Enhanced-Observed Time Difference (E-OTD)}

This technology has been deployed by Cambridge Position Systems. E-OTD operates only on GSM and GPRS networks.

The cell phone sends a signal to the surrounding cell emitters, and the nearest one sends back the signal. The time taken between sending and receiving the wave is analyzed by an external server, which calculates the cell phone position in the network

(www.mobileinfo.com/LocationBasedServices/E_OTD.htm).

This method includes new technology in the handset to assist in locating the unit in a network. Mobile units in an EOTD system are set up to support positioning in a network where BSs are asynchronous.

Theoretically, it takes about 5 seconds to locate a mobile using the E-OTD technique and the accuracy is about $30-50$ meters. Real-world tests have yielded less accurate measurements of about $50-125$ meters [22]. The accuracy of the E-OTD method is a function of the resolution of the time difference measurements, the geometry of the neighbouring base stations, and the signal environment.

E-OTD solutions offer improved performance relative to CID, but require the use of LMUs. This increases the cost and complexity of implementation, as described above. E-OTD also requires a large number of data messages be exchanged to provide location information, and this information must be updated constantly [22].

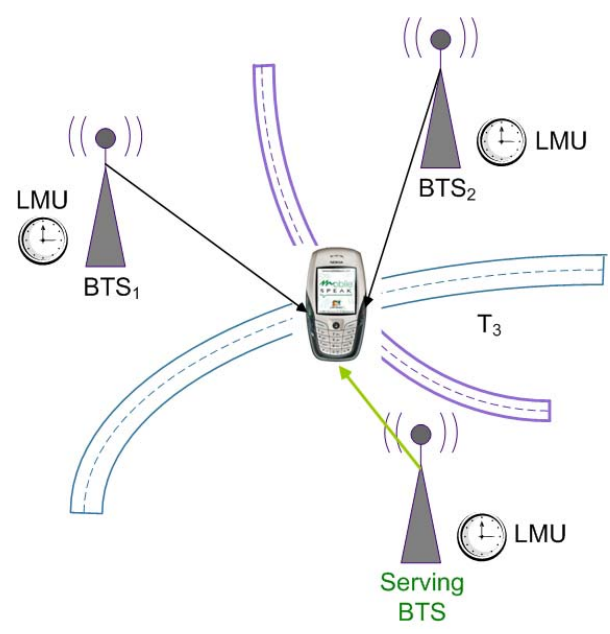

Figure 20. E-OTD Principle.

The message traffic is much greater than that used for AGPS or CID. E-OTD uses more network bandwidth than all other technologies. This technology is vulnerable to accuracy degradation from multipath and signal reflections.

\section{CONCLUSION}

Each of the above described methods has its own advantages and inconvenients. Due to their high resolution capacity in the time domain, time-based positioning algorithms are usually preferred to those involving AOA or RSS measurements. Although these algorithms enable very accurate positioning, they also poses some challenges for practical systems (require installation of dedicated hardware).

The reference point method may provide more accurate positions, without dealing with the above mentioned challenges. Moreover, if RSS is taken as a major parameter to estimate the position of a mobile device, the localization will be easy to design, as most adapters provide RSS measurement.

\section{ACRONYMS}

AP - Access Point - The AP is a device that connects wireless communication devices together to form a wireless network.

APIT - Approximate Point In Triangle Test - APIT employs a novel areabased approach. Anchors divide terrain into triangular regions. A node's presence inside or outside of these triangular regions allows a node to narrow the area in which it can potentially reside.

Autonomous - Term typically used in GPS and wireless A-GPS implementations. A mode of operation in which the MS calculates a position with no aiding from the location server. This mode is also referred to as "standalone" mode, and is commonly the mode in which conventional GPS receivers operate.

A-GPS - Assisted GPS - Aided GPS, or shortened version of Wireless Assisted GPS.

AOA - Angle of Arrival - A location technology that uses special antenna arrays at BTS sites to determine the angle of arrival of handset signals. At 
least two AOA measurements are then combined to identity the position of the handset.

BS - Base Station - In the context of external land surveying, a base station is a GPS receiver at an accurately-known fixed location which is used to derive correction information for nearby portable GPS receivers. In the area of wireless computer networking, a base station is a radio receiver/transmitter that serves as the hub of the local wireless network, and may also be the gateway between a wired network and the wireless network. In radio communications, a base station is a wireless communications station installed at a fixed location and used to communicate.

BTS - Base Transceiver Station - A base station transceiver in the wireless communications infrastructure.

CDMA - Code Division Multiple Access - A high-capacity digital wireless communications technology.

CID - Cell Identification - A location technology that utilizes the location of the BTS to identify caller location.

E-OTD - Enhanced Observed Time Difference - A technique comparing the time an MS signal arrives at different base stations to determine twodimensional position (altitude not determined).

GMLC - Gateway Mobile Location Center - The infrastructure element in $2 \mathrm{G}, 2.5 \mathrm{G}$ and $3 \mathrm{G}$ systems that makes location information available to the location application.

GPRS - GSM Packet Radio System - The packet radio version of GSM communications.

GPS - Global Positioning System - A technique utilizing distance measurements to GPS satellites to determine three-dimensional location (includes altitude).

GSM - Global System for Mobile Communications - Standard for digital mobile telephony originally developed for pan-European use, but now used worldwide.

IPDL - Idle Period Down Link - A signalling method used with OTDOA positioning technology to mitigate the near far problem inherent to UMTS systems.

IR - InfraRed - Infrared radiation is electromagnetic radiation of a wavelength longer than that of visible light, but shorter than that of radio waves. The name means "below red" (from the Latin "infra", "below"), red being the colour of visible light with the longest wavelength.

LMU - Location Measurement Unit - A device used with E-OTD to provide precise timing information for asynchronous networks.

LS - Location Server - The software entity used to calculate position or to provide assistance data or to participate in the positioning process in some other way. Different location technologies require different location server functionality. In report, the LS term will be preceded by a location technology description to indicate the LS supports that type of location technology (e.g., an A-GPS LS is a location server that supports A-GPS technology and therefore provides assistance data and can calculate a final position).

MS - Mobile Station - The term used in GSM and GPRS networks to describe a subscriber's handset or wireless terminal.

MS-Assisted - Term typically used in wireless assisted GPS implementations. A mode of operation in which the MS provides data to the location server to enable the location server to calculated a position.

MS-Based - Term typically used in wireless assisted GPS implementations. A mode of operation in which the MS receives aiding data from the location server to enable the MS to calculate a position.

MSC - Mobile Switching Center - A primary switching hub in 2G, 2.5G and $3 \mathrm{G}$ wireless network.

Multipath fingerprint - A location technology that uses a complex database of stored communication signal images, or "fingerprints" that are each unique to locations throughout the user network. Real-time handset signals are then compared to the stored fingerprints to find a match, which produces a location estimate.

Node B - The term for a base station in a $3 \mathrm{G}$ network.
OTDOA - Observed Time Difference of Arrival - A location technology that compares the time a UE signal arrives at different base stations to determine two-dimensional position (altitude not determined).

P-range - Pseudo range - The distance measurement derived from the elapsed travel time of a GPS signal from a GPS satellite to a GPS receiver.

RF - Radio Frequency - RF refers to that portion of the electromagnetic spectrum in which electromagnetic waves can be generated by alternating current which is fed to an antenna.

RFID - Radio Frequency Identification - RFID is an automatic identification method, relying on storing and remotely retrieving data using devices called RFID tags or transponders. An RFID tag is an object that can be attached to or incorporated into a product, animal, or person for the purpose of identification using radio waves.

RSS - Received Signal Strength - RSS is a measurement of the received radio signal strength (energy integral, not the quality).

RTT - Round Trip Time - A technique utilizing the total round-trip time from an MS to a base station to determine the approximate distance an MS is from a base station.

SAS - Stand-Alone Assisted GPS SMLC - The UMTS network node that may operate the assisted-GPS location server software.

SGSN - Serving GPRS Support Node - The node required in $2.5 \mathrm{G}$ and $3 \mathrm{G}$ systems to provide data support in the core network.

SMLC - Serving Mobile Location Centre - The GSM/GPRS network node that operates the location server software.

SNRC - Serving Network Radio Controller - The UMTS network node that may operate the location server software.

TA - Timing Advance - A technique utilizing the timing advance information applied by the GSM network to determine the approximate distance an MS is from a base station.

TU - Timing Unit - A timing unit required for networks that are not synchronized. Typically uses special GPS receivers to provide absolute timing.

TOA - Time of Arrival - A technique comparing the time an MS signal arrives at different base stations to determine two-dimensional position (altitude not determined)

Trilateration - A technique used to derive location by determining the intersection of hyperbolas derived from the range measurements between a BTS and handset.

TTFF - Time to First Fix - The time elapsed between when a position was requested and when a position was determined. Most commonly applied to GPS and A-GPS technologies, but also applicable for other location technologies.

UE - User Equipment - The term used in UMTS networks to describe a subscriber's handset or wireless terminal.

UMTS - Universal Mobile Telecommunications Systems - UMTS is the third generation $(3 \mathrm{G})$ evolution from $2 \mathrm{G} / 2.5 \mathrm{G}$ networks and since it is based on wideband CDMA radio access technology (WCDMA), it is generally considered synonymous with WCDMA.

WCDMA - Wideband CDMA - IMT-2000 CDMA direct spread standard developed by 3 GPP.

WLAN - Wireless Local Area Network - A WLAN is a wireless local area network, which is the linking of two or more computers without using wires.

Yield - The ratio of the number of successfully calculated positions (meeting specified quality criteria) to the number of attempts to determine a position. A yield of 100 percent means all position attempts resulted in a position calculation within the boundaries of the specified quality criteria. 50 percent yield means only one of every two attempts resulted in a position calculation.

\section{REFERENCES}


[1] Want, R., Hopper, A., Falco, V., and Gibbons, J., "The active badge location system", ACM Transactions on Information Systems, Vol. 10, No. 1, pp.91-102, 1992.

[2] Nakazato, Y., Kanbara, M., and Yokoya, N., "Discreet markers for user localization", Eighth International Symposium on Wearable Computers, ISWC 2004, Volume 1, pp. 172 - 173, 31 October -3 November 2004.

[3] Maeda, M. and Habara, T., "Indoor Localization Methods for Wearable Mixed Reality", Second CREST Workshop on Advanced Computing and Communicating Techniques for Wearable Information Playing, May 24, 2003.

[4] Natarajan, P. and Nahar, G., "Camera Localization methods for Intelligent Room Systems using RF and Stereo Vision Techniques", SPIE 2004 .

[5] Li, Z., Trappe, W., Zhang, Y., and Nath, B., "Robust Statistical Methods for Securing Wireless Localization in Sensor Networks", Fourth International Symposium on Information Processing in Sensor Networks, IPSN 2005.

[6] He, T., Huang, C., Blum, B. M., Stankovic, J. A., and Abdelzaher, T., "Range-Free Localization Schemes for Large Scale Sensor Networks", MobiCom '03, 14-19 September 2003.

[7] Yun, K., and Kim, D., "Robust location tracking using a dual layer particle filter", Pervasive and Mobile Computing, Volume 3, Issue 3, pp. 209 - 232, June 2007.

[8] Ladd, A.M., Bekris, K., Rudys, A., Marceau, G., Kavraki, L.E., and Wallach, D.S., "Robotics-based location sensing using wireless ethernet", ACM MOBICOM, pp. 227 - 238, September 2002.

[9] Haeberlen, A., Flannery, E., Ladd, A.M., Rudys, A., Wallach, D.S., and Kavraki, L.E., "Practical robust localization over large-scale 802.11 wireless networks", ACM MOBICOM, pp. 70 - 84, September 2004.

[10] Yin, J., Yang, Q. and Ni, L., "Adaptive temporal radio maps for indoor location estimation", IEEE PerCom, pp. 85 - 94, March 2005.

[11] Youssef, M., Agrawala, A., and Shankar, A.U., "WLAN location determination via clustering and probability distributions", IEEE PerCom, pp. 143 - 150, March 2003.

[12] Zhao, X., Rautiainen, T., Kalliola, K., and Vainikainen, P., "Path-Loss Models for Urban Microcells at $5.3 \mathrm{GHz}$ ", Antennas and Wireless Propagation Letters, Volume 5, Issue 1, pp. 152 - 154, December 2006.

[13] Abhayawardhana, V.S., Wassell, I.J., Crosby, D., Sellars, M.P., and Brown, M.G., "Comparison of Empirical Propagation Path Loss Models for Fixed Wireless Access Systems", VTC 2005.

[14] Caffery, J. J., "Wireless location in CDMA Cellular Radio Systems", Kluwer Academic, Boston, 2000.

[15] Chen, J.-C., Ting, P., Maa, C.-S., and Chen, J.-T., "Wireless geolocation with TOA/AOA measurements using factor graph and sum-product algorithm", IEEE 60th Vehicular Technology Conference, Volume 5, pp. 3526 - 3529, 26-29 September 2004.

[16] Li, W., and Liu, P., "3D AOA/TDOA emitter location by integrated passive radar/GPS/INS systems", Proceedings of 2005 IEEE International Workshop on VLSI Design and Video Technology, pp. 121 -124, 28-30 May 2005.

[17] Yao, Q., Tan, S.-K., Ge, Y., Yeo, B.-S., and Yin, Q., "An area localization scheme for large wireless sensor networks", IEEE 61st Vehicular Technology Conference, VTC 2005, Volume 5, pp. $2835-$ 2839, 30 May-1 June 2005.

[18] Qi, Y., Kobayashi, H., and Suda, H., "Analysis of wireless geolocation in a non-line-of-sight environment", IEEE Transactions on Wireless Communications, Volume 5, Issue 3, pp. 672 - 681, March 2006.

[19] Lee, J.-Y., and Scholtz, R.A., "Ranging in a dense multipath environment using an UWB radio link", IEEE Transactions on Selected Areas in Communications, vol. 20, no. 9, pp. 1677 - 1683, December 2004.

[20] Adams, J.C., Gregorwich, W., Capots, L., and Liccardo, D., "Ultrawideband for navigation and communications", Proceedings IEEE Aerospace Conference, vol. 2, pp. 785 - 792, March 2001.

[21] "Synchronous vs. Asynchronous Deployment of WCDMA - Effect on Communication System Performance", QUALCOMM, September 2001.

[22] "An Examination of U-TDOA and Other Wireless Location Technologies: Their Evolution and Their Impact on Today's Wireless Market", TruePosition, 2004.
[23] Angelides, J., “A practical look at A-GPS”, Global Telecoms Business: $\mathrm{CEO}$ and CFO Guide to Broadband and Wireless, January/February 2004.

[24] Dedes, G., and Dempster, A.G., "Indoor GPS Positioning : Challenges and Opportunities", Vehicular Technology Conference, vol. 1, pp. 412 415, 28-25 September 2005.

[25] Niculescu, D., and Nath, B., "Ad Hoc Positioning System (APS) Using AoA", 2003.

[26] Qi, Y., Kobayashi, H., and Suda, H., "Analysis of Wireless Geolocation in a Non-Line-of-Sight Environment", IEEE Transactions on wireless communications, vol. 5, no. 3, March 2006. 